\title{
A UTILIZAÇÃO DA BIBLIOMETRIA NA ANÁLISE DO REFERENTE CONFORTO
}

\author{
Danielle Copello Vaz ${ }^{1}$, Carlos Roberto Lyra da Silva², Nébia Maria Almeida de Figueiredo ${ }^{3}$
}

RESUMO: A partir do Estado da Arte desenvolvido durante a elaboração da tese "Conceito de conforto na perspectiva de clientes e enfermeiras em unidades de internação hospitalar", foi possível detectar a precária quantidade de produtos científicos acerca do conforto como objeto de investigação em Enfermagem. Diante do exposto, este trabalho tem como objetivo identificar as formas de utilização da palavra conforto na produção científica nacional e internacional. Trata-se de um estudo bibliométrico de abordagem quantitativa. Dos 535 resumos encontrados, 142 pertenciam à Enfermagem. Os resultados revelam que houve um aumento do número de produções a partir da década de 90, principalmente na região sudeste, e que a maioria das publicações foi em português. A palavra conforto não é reconhecida como um descritor; sendo assim, o descritor que mais foi utilizado pelos autores em seus estudos foi cuidado. Conclui-se que houve um aumento expressivo da produção científica na área da Enfermagem.

PALAVRAS-CHAVE: Bibliometria; Cuidados de conforto; Enfermagem.

\section{USE OF BIBLIOMETRY FOR COMFORT REFERENT ANALYSIS}

\begin{abstract}
From the State of the Art, developed during the preparation of the thesis "Concept of comfort from the perspective of clients and nurses in hospital inpatient units," it was possible to detect the precarious quantity of scientific products from the comfort as an object of research in Nursing. Given the above, this paper aims to identify ways to use the word comfort in the international and national scientific production. This is a bibliometric study of quantitative approach. Of the 535 abstracts found, 142 belonged to Nursing. The results show that there is an increased number of productions from the $90 \mathrm{~s}$, mainly in the Southeast of Brazil and most publications were in Portuguese. The word comfort is not recognized as a descriptor, so the descriptor that was most used by the authors in their studies was caring. It is concluded that there was a significant increase of scientific production in nursing.
\end{abstract}

KEYWORDS: Bibliometrics; Comfort care; Nursing.

\section{LA UTILIZACIÓN DE LA BIBLIOMETRÍA EN EL ANÁLISIS DEL REFERENTE CONFORTACIÓN}

RESUMEN: Con base en Estado del Arte, desarrollado durante la elaboración de la tesis "Concepto de confortación en la perspectiva de clientes y enfermeras en unidades de internación hospitalar", fue posible detectar la precaria cuantidad de productos científicos acerca de la confortación como objeto de investigación en Enfermería. Delante del expuesto, este trabajo tiene como objetivo identificar las formas de utilización de la palabra confortación en la producción científica nacional y internacional. Es un estudio bibliométrico de abordaje cuantitativo. De los 535 resúmenes encontrados, 142 pertencían a la Enfermería. Los resultados refieren que hubo un aumento del número de producciones a partir de la década de 90, principalmente en la región sudeste y la mayoría de las publicaciones estaba en portugués. La palavra confortación no es reconocida como un descriptor, siendo así, el descriptor que fue más utilizado por los autores en sus estudios fue cuidado. Se concluye que hubo un aumento expresivo de la producción científica en el área de la enfermería. PALABRAS CLAVE: Bibliometría; Cuidados de confortación; Enfermería.

\footnotetext{
${ }^{1}$ Enfermeira.

${ }^{2}$ Enfermeiro. Doutor em Enfermagem. Professor Adjunto do Departamento de Enfermagem Fundamental da Escola de Enfermagem Alfredo Pinto-EEAP/UNIRIO.

${ }^{3}$ Enfermeira. Doutora em Enfermagem. Professor Titular do Departamento de Enfermagem Fundamental da EEAP/UNIRIO.
}

\author{
Autor correspondente: \\ Danielle Copello Vaz \\ Universidade Federal do Estado do Rio de Janeiro \\ Av. Pasteur, 296 - 22290-240 - Urca-RJ-Brasil \\ E-mail: dani_copello@hotmail.com
}

Recebido: 16/06/10 Aprovado: 10/10/10 


\section{INTRODUÇÃO}

A literatura de Enfermagem demonstra que, desde o início da profissão até os dias atuais, o conforto tem sido uma meta do cuidado e um conceito presente em toda a sua história ${ }^{(1-2)}$. O conforto, etimologicamente, se origina do latim confortare, que significa fortificar, certificar, corroborar, conceder, consolar, aliviar, assistir, ajudar e auxiliar ${ }^{(3)}$.

Sabe-se que a Enfermagem evoluiu através dos séculos. Nas últimas décadas, o aprofundamento e o desenvolvimento da profissão fizeram com que a enfermeira passasse por um processo de reflexão teórica, não obstante ter que dominar cada vez mais a evolução tecnológica para, a partir dela, corroborar sua prática de assistir e de cuidar de clientes, sobretudo os criticamente enfermos, como é o caso dos cuidados em unidade de terapia intensiva. Além disso, houve uma ampliação, diversificação e acúmulo de tarefas, o que coloca a enfermeira na condição de responsável pela supervisão, administração e gerenciamento dos cuidados ${ }^{(4)}$. Diante dessas mudanças, acredita-se que o profissional passou a dispor de pouco tempo para estar em contato direto com o paciente, sendo essa função suprida pelos auxiliares e técnicos e, o que é pior, os atos e as representações desses profissionais são substituídos pelas tecnologias duras.

Nesse sentido, o conforto pode deixar de ser uma meta primordial na assistência prestada pelo enfermeiro, passando a ser uma meta secundária. No entanto, esse referente (conforto) é amplamente citado nos discursos dos profissionais de saúde, em particular, os profissionais de enfermagem, seja no plano de ação ou de investigação.

A partir do Estado da Arte desenvolvido durante a elaboração da tese de doutorado "Conceito de conforto na perspectiva de clientes e enfermeiras em unidades de internação hospitalar"(5), foi possível detectar a precariedade na quantidade de produtos científicos, sobretudo no âmbito nacional, acerca do conforto como objeto de investigação em Enfermagem.

Desta forma, acredita-se que, mesmo sendo um referente propalado no discurso da Enfermagem, parece que ele não tem despertado interesse como objeto de investigação científica.

Considerando a jovialidade da Enfermagem como disciplina/conhecimento, seus saltos ainda estão intimamente ligados ao seu fazer, carecendo ainda, de um espírito mais questionador que possa, por meio da produção de conhecimento, buscar evidências de que o cuidado e o conforto são também objetos reais de investigações capazes de contribuir para construção de uma enfermagem ciência ${ }^{(6: 770)}$.

Portanto, o estudo é relevante para a Enfermagem, pois se acredita que poderão emergir reflexões sobre o seguinte aspecto: de que forma a palavra conforto está sendo utilizada nas investigações científicas?

Assim, esse estudo tem como objetivo identificar as formas de utilização da palavra conforto na produção científica nacional e internacional.

\section{METODOLOGIA}

Trata-se de um estudo bibliométrico de natureza quantitativa. A bibliometria pode ser definida como:

O estudo dos aspectos quantitativos da produção, disseminação e uso da informação registrada. A bibliometria desenvolve padrões e modelos matemáticos para medir esses processos, usando seus resultados para elaborar previsões e apoiar tomadas de decisões ${ }^{(7: 134)}$.

De modo geral, o princípio da bibliometria é analisar a atividade científica ou técnica, pelo estudo quantitativo das publicações, e o seu principal objetivo é o desenvolvimento de indicadores cada vez mais confiáveis. Os indicadores podem ser definidos como os parâmetros utilizados nos processos de avaliação de qualquer atividade ${ }^{(8)}$.

Para o desenvolvimento deste trabalho foi utilizado o site da Biblioteca Virtual em Saúde (BVS), onde foi feita uma pesquisa com a palavra conforto. Foi disponibilizada como resultado uma lista do banco de dados, da qual foram selecionadas as seguintes bases: BDENF (Base de Dados Nacionais da Enfermagem) e LILACS (Literatura Latino-Americana e do Caribe em Ciências da Saúde). Foi possível identificar, ao todo, 98 e 535 resumos em cada base, respectivamente. As bases de dados selecionadas não disponibilizam todos os artigos na íntegra. Logo, todas as informações obtidas neste estudo foram extraídas dos resumos.

Para organizar os dados dos 633 resumos, foram estabelecidas as seguintes variáveis: base de dados (BDENF e LILACS); área do conhecimento (Enfermagem, Medicina, Psicologia, entre outras); todos os autores; periódico; descritores utilizados no resumo do artigo; ano e idioma de publicação. Todas essas informações foram armazenadas numa planilha.

A fim de obter os dados exclusivamente da 
área da Enfermagem, foram estabelecidos previamente os critérios de inclusão e exclusão do estudo. Critérios de inclusão: resumos com a palavra enfermagem no título, no corpo do resumo ou nos descritores; artigos que foram publicados em revistas especializadas na área de enfermagem; dissertações de mestrado e teses de doutorado. Os resumos que foram excluídos não estavam de acordo com os critérios anteriores ou estavam em duplicidade na mesma base de dados, ou em ambas as bases.

Após a leitura de todos os resumos e aplicação dos critérios de inclusão e exclusão, foram selecionados 142 resumos. A partir desse corpus foram analisadas todas as variáveis propostas no estudo.

\section{RESULTADO E DISCUSSÃO}

\section{Base de dados}

Um problema detectado no decorrer da pesquisa foi a duplicidade do mesmo resumo na mesma base de dados, ou em ambas. Logo, para se obter uma amostra o mais fidedigna possível, esses artigos foram excluídos do estudo.

A amostra inicial da BDENF era de 98 resumos, sendo que, destes, 13 estavam em duplicidade na base de dados, 74 estavam repetidos em ambas as bases e 11 eram exclusivos da BDENF.

A amostra inicial da LILACS era de 535 resumos. No entanto, apenas 131 resumos pertenciam à Enfermagem. Deste quantitativo, apenas 57 eram exclusivos da LILACS.

Logo, a amostra final da área de conhecimento de Enfermagem ficou com o quantitativo de 142 artigos (Gráfico 1). A Figura 1 indica a distribuição da amostra final.

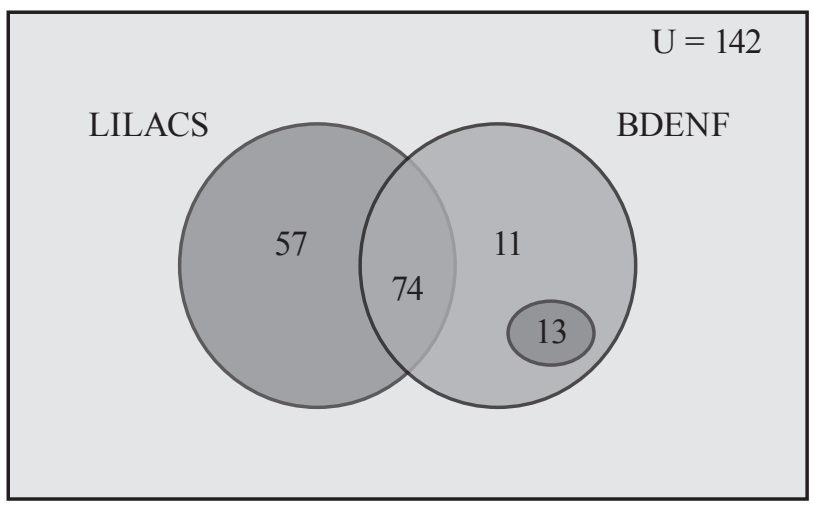

Figura 1 - Distribuição dos resumos pertencentes à área de Enfermagem - BDENF e LILACS

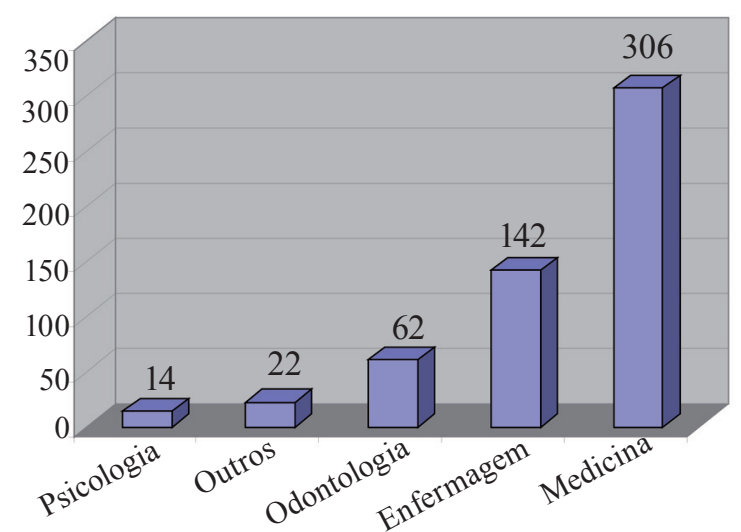

Gráfico 1 - Distribuição das áreas de conhecimento de ambas as bases de dados (número de artigos x área de conhecimento) - BDENF e LILACS

Importante mencionar que apenas a área de conhecimento Enfermagem foi analisada em relação a duplicidade do resumo na mesma base de dados, ou em ambas. Desta forma, as outras áreas de conhecimento podem estar com o valor superestimado, uma vez que o mesmo artigo pode estar sendo contabilizado duas ou mais vezes na mesma base de dados. Mesmo assim, a presença da investigação em Enfermagem mostrou-se bastante significativa, pois de um total de 546 referências, 142 são da Enfermagem, o que representa $26 \%$ da amostra estudada. No entanto, esse quantitativo poderia ser ainda mais expressivo, caso os autores tivessem a preocupação de colocar a palavra enfermagem no título, resumo ou nos descritores dos trabalhos. Desta forma, toda a produção poderia ser mensurada de uma forma mais fidedigna e, por conseguinte, fortaleceria ainda mais a produção de conhecimento da Enfermagem.

\section{Categorização dos Descritores}

Todos os descritores utilizados pelos autores dos artigos foram analisados, sendo totalizados 672 descritores.

O critério para inclusão de um descritor em uma categoria foi a utilização de uma determinada palavra específica. Deste modo, comparou-se a lista de termos da variável e da categoria, e definiu-se então o termo que melhor se enquadrasse no critério; a partir deste resultado, avaliou-se o termo pertencente à categoria.

Foram definidas 7 categorias: assistência (30), cuidado (123), condição clínica/patologia (89), enfermagem (61), hospital (22), paciente (22) e relação (26). 
Devido à grande diversidade dos descritores, conseguimos categorizar apenas 55\% da amostra (373 descritores).

Percebemos, com a análise dessa variável, que conforto está diretamente relacionado com a questão do cuidado, pois este foi o descritor mais mencionado pelos autores.

\section{Idioma de Publicação}

O idioma com maior destaque foi português com $124(87,32 \%)$ artigos, seguido do espanhol com $17(11,98 \%)$ e somente $1(0,7 \%)$ no inglês. Caso as publicações na língua inglesa fossem mais expressivas, um número maior de pessoas em nível mundial teria acesso ao material, favorecendo, desta forma, o intercâmbio global de conhecimentos. No entanto, houve destaque das publicações na língua portuguesa, restringindo a disseminação do conhecimento.

\section{Análise cronológica das publicações}

O período encontrado na análise cronológica está compreendido entre o ano de 1964 até o ano de 2007. Como pode ser observado no gráfico 2 , apenas $1(0,7 \%)$ trabalho na década de sessenta, $4(2,8 \%)$ na década de setenta, $12(8,5 \%)$ na década de oitenta, 43 (30,3\%) na década de noventa e $81(57 \%)$ entre os anos 2000 e 2007. Apenas $1(0,7 \%)$ resumo não possuía o ano de publicação.

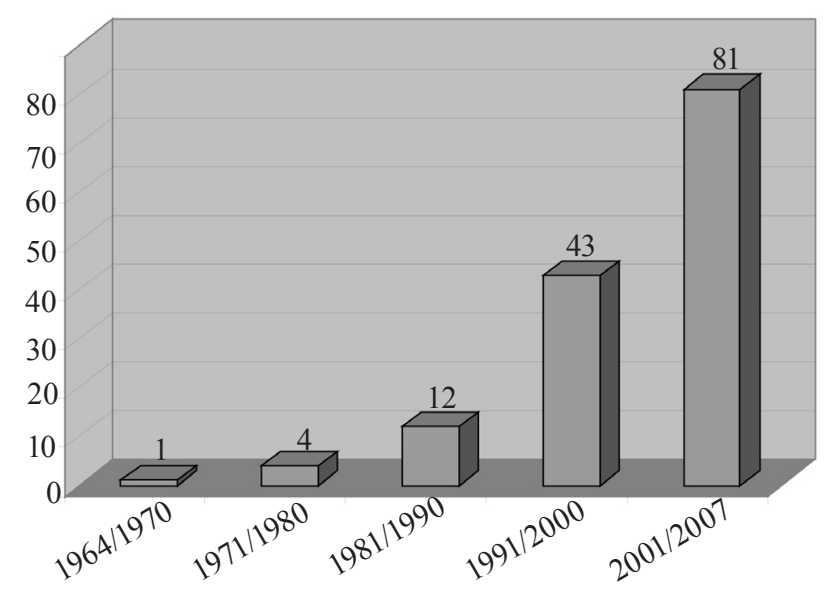

Gráfico 2 - Evolução cronológica das publicações (número de artigos $\mathrm{x}$ período) - BDENF LILACS

Podemos observar que houve um aumento quantitativo de produções científicas na área de Enfermagem. Este gráfico é justificado devido a uma série de fatos relevantes que ocorreram nas últimas décadas para o incentivo e a incorporação da pesquisa pela Enfermagem.

Em 1972, foi criado o primeiro Curso de Mestrado do Brasil, na Escola de Enfermagem Anna Nery da Universidade Federal do Rio de Janeiro (EEAN/ UFRJ). No início dos anos 80 , houve a criação do primeiro Curso de Doutorado em Enfermagem no Brasil e na América Latina, na Escola de Enfermagem da USP, em conjunto com a Escola de Enfermagem de Ribeirão Preto (EERP) da USP (doutorado interunidades $)^{(9)}$. Outro aspecto importante que propiciou o aumento da produção científica ocorreu nos anos 90, que foi a constituição de grupos de pesquisa e núcleos de estudos em uma área temática, congregando pessoas em diferentes estágios de formação científica (alunos de iniciação científica, aperfeiçoamento, mestrado e doutorado e pesquisadores) ${ }^{(10)}$. No entanto, acredita-se que o principal responsável por esse aumento da produção científica nacional do sistema de pós-graduação que, por meio da CAPES, tem priorizado o número de artigos publicados para conceituar os programas nacionais ${ }^{(11)}$.

\section{Região do periódico}

Devido à grande diversidade de periódicos encontrados, foi estabelecido um critério a fim de organizar melhor os dados. Para um periódico ser contabilizado, precisaria ter, no mínimo, três publicações de artigos. Desta forma, selecionamos apenas 11 periódicos, sendo $1(9,1 \%)$ da região centro-oeste, 3 $(63,6 \%)$ da região sul e $7(27,3 \%)$ da região sudeste. A região com maior destaque foi a região sudeste, com 41 (54,7\%) artigos publicados, seguida da região sul, com 22 (29,3\%), e centro-oeste, com 12 (16\%).

Em relação às dissertações de mestrado e teses de doutorado, todas foram contabilizadas, uma vez que a quantidade não foi tão expressiva. Acredita-se que este fato tenha ocorrido devido, ainda, a um número muito restrito de dissertações e teses indexadas na íntegra nas bases de dados.

Foram encontradas 23 dissertações de mestrado, sendo $2(8,7 \%)$ da região nordeste, $5(21,7 \%)$ do sul e $16(69,6 \%)$ do sudeste. Em relação às teses de doutorado, foram encontradas apenas 6 , todas da região sudeste.

Podemos observar que a maioria das publicações ocorre na região sudeste. Esse fato se explica pelo maior número de instituições de ensino e pesquisa 
nessa região. As instituições que possuem cursos de pós-graduação concentram a maior parte da produção científica brasileira $^{(12-13)}$. Dos trinta e cinco cursos de pós-graduação na área de Enfermagem, recomendados e reconhecidos pela CAPES, 18 (51,43\%) estão localizados na região sudeste.

\section{Autores com maior número de publicações}

As autoras com o maior destaque no número de publicações são Eloita Pereira Neves com 11 $(52,4 \%)$ artigos, Fernanda Carneiro Mussi com 7 $(33,3 \%)$ artigos e Nébia Maria Almeida de Figueiredo com $3(14,3 \%)$ artigos.

Observamos que o número de pesquisadores envolvidos com a temática conforto ainda é muito restrito. Dos 142 resumos selecionados neste estudo, apenas 21 (14,79\%) pertencem a 3 autoras. Percebese que o tema em questão desperta pouco interesse dos autores, uma vez que foram encontradas apenas 3 autoras com mais de 2 publicações. Desta forma, acredita-se que o conforto, embora seja amplamente mencionado nos estudos, é pouco aprofundado nas investigações.

\section{CONCLUSÃo}

O estudo permitiu um panorama da produção científica brasileira sobre o conforto. A análise bibliométrica permite afirmar que existe um crescimento notável no número de trabalhos sobre esse tema, principalmente a partir da década de 90 . Esse fato está relacionado ao aumento expressivo de pesquisadores na área da Enfermagem, decorrente da política de qualificação profissional e do novo papel assumido pela academia.

A bibliometria é utilizada para observar o conhecimento registrado, isto é, publicado. Logo, um viés encontrado neste estudo é que nem tudo que é produzido é publicado. Geralmente, os trabalhos publicados são mais de profissionais vinculados a universidade ou centro de pesquisa do que aqueles dedicados à prática. Sendo assim, o mapeamento ficou restrito aos artigos encontrados nas bases. No entanto, acredita-se que os artigos que foram publicados nas revistas indexadas nas bases de dados possuem qualidade e relevância asseguradas.

Os cursos de pós-graduação estão diretamente associados à construção do conhecimento científico, uma vez que são responsáveis pela formação de profissionais qualificados, pesquisadores e produção de novos conhecimentos. No entanto, observamos uma distribuição geográfica heterogênea da produção científica no nosso país, na qual o sudeste possui a maior concentração de universidades, sendo por isso responsável pela maior produção de pesquisas do país.

\section{REFERÊNCIAS}

1. McIlveenn KH, Morse J. The role of comfort in nursing care: 1900-1980. Clin Nurs Res. 1995;4(3):127-48.

2. Mussi FC. Padecendo a perda da espontaneidade da ação: o desconforto vivenciado por homens que sofreram infarto agudo do miocárdio [tese]. São Paulo(SP): Universidade de São Paulo; 2000.

3. Rosa LM, Mercês NNA, Santos VEP, Radünz V. As faces do conforto: visão de enfermeiras e pacientes com câncer. Rev Enferm UERJ. 2008;16(3):404-10.

4. Mussi FC. Conforto e lógica hospitalar: análise a partir da evolução histórica do conceito na enfermagem. Acta Paul Enferm 2005;18(1):72-81.

5. Silva CRL. Conceito de conforto na perspectiva de clientes e enfermeiras em unidades de internação hospitalar [tese]. Rio de Janeiro (RJ): Universidade Federal do Rio de Janeiro; 2008.

6. Silva CRL, Carvalho W, Figueiredo NMA. Aspectos epistemológicos do cuidado e conforto como objeto de conhecimento em enfermagem. Cogitare Enferm. 2009;14(4):762-72.

7. Marcias-Chapula CA. O papel da informetria e da cienciometria e sua perspectiva nacional e internacional. Ciênc Inf. 1998;27(2):134-40.

8. Hayashi MCPI, Hayashi CRM, Silva MR, Lima MY. Um estudo bibliométrico da produção científica sobre a educação jesuíta no Brasil colonial. Biblios 2007;27(1):1-18.

9. Salles EB, Barreira IA. Formação da comunidade científica de enfermagem no Brasil. Texto \& Contexto Enferm. 2010;19(1): 137-46.

10. Arruda EPN. A pesquisa no cotidiano da enfermagem: pesquisa em grupos, núcleos e parcerias. In: Seminário Nacional de Pesquisa em Enfermagem - a pesquisa no cotidiano da enfermagem, 1995. p. 44-60 Ribeirão Preto; Brasil. 1995.

11. Souza PR. O crescimento da produção científica. Folha 
de São Paulo. 2002; Nov 06; Sect A3.

12. Figueira I, Jacques R, Leta J. A comparison between domestic and international publications in Brazilian psychiatry. Scientometrics 2003;56(3):317-27.

13. Bressan RA, Gerolin J, Mari JJ. The modest but growing Brazilian presence in psychiatry, psychobiological and mental health research: assessment of the 1998-2001 period. Braz J Med Biol Res. 2005;38:64959. 